\title{
II. Geburtshülflich-gynäkologische Mitteilungen aus Medizinischen Gesellschaften und Vereinen
}

Medizinische Sektio der schlesischen Gesellschaît für vaterländische Kultur in Breslau.

18. 1. (Berl. k¹/8. Woch. 6. II. S. 43õ.) Heimann: 5 Jahre Strahlen-therapie.

Aussprache: Küstner, L. Fraenkel, Marx, Silberberg, Henke, Jadas-s $\sigma$ hn, Heimann.

72

Buchanzeigen.

Verein für wissenschaftliehe Heilkunde zu Königsberg i. Pr.

14. I. (Berl. klia Woch. 6. V. 438.) Sobotta: Eineiige Zwilünge und' Polyembryonie. - Sachs:

Über Nierenleiden in der Schwangerschaft. - Derselbe, Mißbildung innerer Genitalien. 8. I.

Ebenda. S. 438.

Medizinische Gesellschaft zu Kiel.

14. II. (Berl. klin. Woch. 27 V. S. 510.) Stoßckel: Demonstration eines Falles von Maydl'scher

Operation bei tuberkulöser Schrumpfblase.

Buchanzeigen.

O. Küstner, Kurzes Lehrbuch der Gynäkologie. Bearbeitet von E. Bumm, A. Döderlem, B.

Krönig, C. Me,nge, herausgegeben von O. Küstner. TV. П3u überarbeitete Auflage. Jena 1917.

G. Fischer.

$\mathrm{Da}$ dieses Lehrbuch trotz der Ungunst der Ze.it neu überarboitet werden konnte, ist ein um so stolzerer Bew ,is für deutsches wissenschaftliches Strebon, als alle Bet $\theta$ iligten S $\theta$ it Kriegsbeginn sich gleichzeitig in bchwerster Heeresarbeit betätigt haben. Sie haben es mit der Überarbeitang ihrer Aı-teil $\theta$ seh $\Gamma$ sehr ernst genommen : vielfach sind Abstrich $\theta$, Vielfach Zusätze vorgenommen worden. Domneuesten Kapitel der Gynäkologie, der Strahlen-behandlung, ist Von Fritz Heimann eine ausführliehe Darstellung gewidmet. Unter dem Einfluß dieses Verfahrens ist die Behandlung des Myom und des Karzinom umgeechrieben. Eine kurze, sehr zutreffende Darstellung haben Thermo-, Licht- und Balneotherapie erhalten. Die Bilder sind ergänzt, das Sachregister mit besonderer Sorgf'”3/8 avsführlicher gestaltet.

Ref. ist auch in dieser neuon Auflage mit vollem Interesse den Aus-führungen der einzelnen Abschnitte naebgegangen; er hat sich damit einen gleichbleibenden Genuß verschafft! Zuversichtlich wird dieses Lehrbuch dem jungen Nachwuchs der Gynäkologen, zumal denen, welehe als Fold-unterärzt $\theta$ gerade zu gynäkologisohen Studien nur eine sehr besehränkte Muß $\beta$ finden, dann, worm sie sich friedlicher Arbeit widmen sollen, ein gesuchter und Vortrefflicher Ratgeber, den Praktikern jedes Alters a bar ein sehr nützlichor Weggenosse werden. Dann werden wir bald die Heraus-geb $\beta r$ mit der VII. Auflage beschäftigt sehen; sie werden den staunens-werten Ergebnissen, die wir gerade auf dem Gebiet der Gynäkologie trotz alledem gerade in diesen letzten Jahren - also während der Drucklegung der VI. Auflage -reifßn sahen, Reehnung tragen müssen. Aus ihrer siolzen Fülle nennen wir bier - um nur diese hier zu erwähnen - die Bumm-sch $\theta$ Collifixu $\Gamma$ als Prolapsoperation, die Stoeckels $\beta$ hs Inkontinenz- 
operation, die Ausbildung der Ureterenchirurgie, die neuen N3/4rkosen-mt,thoden, die neuen Fortschritte auf dem Gebiet der Strahlen-Tiefentherapi $\theta$. Auch das alte, noch nicht restlos gelöst $\theta$ Problem der Vorfalloperation hat d $\backslash$ urch das zu neuer Bedeutung von 0. v. Herff herausgßarb $\theta i t \theta t e ~ N e u g e b a u e r-L e f o r t s G h .9$ Verfahren und die Ho/meiersche Modifikation eine sehr beachtenswerte Förderung erfahren. Über diese und andere Anregungen werden dann Nachprüfungen vorlîegen, über welch $\beta$ wir ein maßg $\theta$ bendes Urteil gerade von den führenden fünf Heraus-gebern wohl erwarten dürfen! Sia haben uns in der vorliogenden VI. Auflage 